\title{
Erratum to: Quantum Yang-Mills field theory
}

\author{
Marco Frasca
}

Via Erasmo Gattamelata, 3, 00176 Rome, Italy

Original article: Eur. Phys. J. Plus (2016) 132: 38, DOI: 10.1140/epjp/i2017-11321-4

Received: 10 May 2017

Published online: 31 May 2017 - (C) Società Italiana di Fisica / Springer-Verlag 2017

After publication, the author has realized that a couple of indexes were not properly set in the equations for the 1-point function (eqs. (60) and (61)) whose correct version is as follows:

$$
\begin{aligned}
& \partial^{2} G_{1 \nu}^{(j) a}(x)+g f^{a b c}\left(\partial^{\mu} G_{2 \mu \nu}^{(j) b c}(x, x)+\partial^{\mu} G_{1 \mu}^{(j) b}(x) G_{1 \nu}^{(j) c}(x)-\partial_{\nu} G_{2 \mu}^{(j) \mu b c}(x, x)-\partial_{\nu} G_{1 \mu}^{(j) b}(x) G_{1}^{(j) \mu c}(x)\right) \\
& +g f^{a b c} \partial^{\mu} G_{2 \mu \nu}^{(j) b c}(x, x)+g f^{a b c} \partial^{\mu}\left(G_{1 \mu}^{(j) b}(x) G_{1 \nu}^{(j) c}(x)\right) \\
& +g^{2} f^{a b c} f^{c d e}\left(G_{3 \mu \nu}^{(j) \mu b d e}(x, x, x)+G_{2 \mu \nu}^{(j) b d}(x, x) G_{1}^{(j) \mu e}(x)\right. \\
& +G_{2 \nu \rho}^{(j) e b}(x, x) G_{1}^{(j) \rho d}(x)+G_{2 \mu \nu}^{(j) d e}(x, x) G_{1}^{(j) \mu b}(x) \\
& \left.+G_{1}^{(j) \mu b}(x) G_{1 \mu}^{(j) d}(x) G_{1 \nu}^{(j) e}(x)\right)=g f^{a b c}\left(\partial_{\nu} P_{2}^{(\varepsilon) b c}(x, x)+\partial_{\nu}\left(\bar{P}_{1}^{(\varepsilon) b}(x) P_{1}^{(\varepsilon) c}(x)\right)\right)+j_{\nu}^{a}
\end{aligned}
$$

and then, for $j=0$,

$$
\begin{aligned}
& \partial^{2} G_{1 \nu}^{a}(x)+g f^{a b c}\left(\partial^{\mu} G_{2 \mu \nu}^{b c}(0)+\partial^{\mu} G_{1 \mu}^{b}(x) G_{1 \nu}^{c}(x)-\partial_{\nu} G_{2 \mu}^{\nu b c}(0)-\partial_{\nu} G_{1 \mu}^{b}(x) G_{1}^{\mu c}(x)\right) \\
& +g f^{a b c} \partial^{\mu} G_{2 \mu \nu}^{b c}(0)+g f^{a b c} \partial^{\mu}\left(G_{1 \mu}^{b}(x) G_{1 \nu}^{c}(x)\right) \\
& +g^{2} f^{a b c} f^{c d e}\left(G_{3 \mu \nu}^{\mu b d e}(0,0)+G_{2 \mu \nu}^{b d}(0) G_{1}^{\mu e}(x)\right. \\
& +G_{2 \nu \rho}^{e b}(0) G_{1}^{\rho d}(x)+G_{2 \mu \nu}^{d e}(0) G_{1}^{\mu b}(x) \\
& \left.+G_{1}^{\mu b}(x) G_{1 \mu}^{d}(x) G_{1 \nu}^{e}(x)\right)=g f^{a b c}\left(\partial_{\nu} P_{2}^{b c}(0)+\partial_{\nu}\left(\bar{P}_{1}^{b}(x) P_{1}^{c}(x)\right)\right)
\end{aligned}
$$

This indexes problem was also present in the equations for the 2-point function of the gluon (eqs. (62) and (63)), where a term was also omitted. These should read as follows:

$$
\begin{aligned}
& \partial^{2} G_{2 \nu \kappa}^{(j) a m}(x-y)+g f^{a b c}\left(\partial^{\mu} G_{3 \mu \nu \kappa}^{(j) b c m}(x, x, y)+\partial^{\mu} G_{2 \mu \kappa}^{(j) b m}(x-y) G_{1 \nu}^{(j) c}(x)+\partial^{\mu} G_{1 \mu}^{(j) b}(x) G_{2 \nu \kappa}^{(j) c m}(x-y)\right. \\
& \left.-\partial_{\nu} G_{3 \mu \kappa}^{(j) \mu b c m}(x, x, y)-\partial_{\nu} G_{2 \mu \kappa}^{(j) b m}(x-y) G_{1}^{(j) \mu c}(x)-\partial_{\nu} G_{1 \mu}^{(j) b}(x) G_{2 \kappa}^{(j) \mu c m}(x-y)\right) \\
& +g f^{a b c} \partial^{\mu} G_{3 \mu \nu \kappa}^{(j) b c m}(x, x, y)+g f^{a b c} \partial^{\mu}\left(G_{2 \mu \kappa}^{(j) b m}(x-y) G_{1 \nu}^{(j) c}(x)\right)+g f^{a b c} \partial^{\mu}\left(G_{1 \mu}^{(j) b}(x) G_{2 \nu \kappa}^{(j) c m}(x-y)\right) \\
& +g^{2} f^{a b c} f^{c d e}\left(G_{4 \mu \nu \kappa}^{(j) \mu b d e m}(x, x, x, y)+G_{3 \mu \nu \kappa}^{(j) b d m}(x, x, y) G_{1}^{(j) \mu e}(x)+G_{2 \mu \nu}^{(j) b d}(x, x) G_{2 \kappa}^{(j) \mu e m}(x-y)\right. \\
& +G_{3 \nu \rho \kappa}^{(j) a c m}(x, x, y) G_{1}^{(j) \rho b}(x)+G_{2 \nu \rho}^{(j) e b}(x, x) G_{2 \kappa}^{(j) \rho d m}(x-y)+G_{2 \nu \rho}^{(j) d e}(x, x) G_{2 \kappa}^{(j) \rho b m}(x-y)+G_{1}^{(j) \mu b}(x) G_{3 \mu \nu \kappa}^{(j) d e m}(x, x, y) \\
& \left.+G_{2 \kappa}^{(j) \mu b m}(x-y) G_{1 \mu}^{(j) d}(x) G_{1 \nu}^{(j) e}(x)+G_{1}^{(j) \mu b}(x) G_{2 \mu \kappa}^{(j) d m}(x-y) G_{1 \nu}^{(j) e}(x)+G_{1}^{(j) \mu b}(x) G_{1 \mu}^{(j) d}(x) G_{2 \nu \kappa}^{(j) e m}(x-y)\right) \\
& \left.=g f^{a b c}\left(\partial_{\nu} K_{3 \kappa}^{(j \varepsilon) b c m}(x, x, y)+\partial_{\nu}\left(\bar{P}_{1}^{(\varepsilon) b}(x) K_{2 \kappa}^{(j \varepsilon) c m}(x, y)\right)\right)+\partial_{\nu}\left(\bar{K}_{2 \kappa}^{(j \varepsilon) b m}(x, y) P_{1}^{(\varepsilon) c}(x)\right)\right)+\delta_{a m} g_{\nu \kappa} \delta^{4}(x-y)
\end{aligned}
$$

\footnotetext{
${ }^{a}$ e-mail: marcofrasca@mclink.it
} 
and, for $j=0$,

$$
\begin{aligned}
& \partial^{2} G_{2 \nu \kappa}^{a m}(x-y)+g f^{a b c}\left(\partial^{\mu} G_{3 \mu \nu \kappa}^{b c m}(0, x-y)+\partial^{\mu} G_{2 \mu \kappa}^{b m}(x-y) G_{1 \nu}^{c}(x)+\partial^{\mu} G_{1 \mu}^{b}(x) G_{2 \nu \kappa}^{c m}(x-y)\right. \\
& \left.-\partial_{\nu} G_{3 \mu \kappa}^{\mu b c m}(0, x-y)-\partial_{\nu} G_{2 \mu \kappa}^{b m}(x-y) G_{1}^{\mu c}(x)-\partial_{\nu} G_{1 \mu}^{b}(x) G_{2 \kappa}^{\mu c m}(x-y)\right) \\
& +g f^{a b c} \partial^{\mu} G_{3 \mu \nu \kappa}^{b c m}(0, x-y)+g f^{a b c} \partial^{\mu}\left(G_{2 \mu \kappa}^{b m}(x-y) G_{1 \nu}^{c}(x)\right)+g f^{a b c} \partial^{\mu}\left(G_{1 \mu}^{b}(x) G_{2 \nu \kappa}^{c m}(x-y)\right) \\
& +g^{2} f^{a b c} f^{c d e}\left(G_{4 \mu \nu \kappa}^{\mu b d e m}(0,0, x-y)+G_{3 \mu \nu \kappa}^{b d m}(0, x-y) G_{1}^{\mu e}(x)+G_{2 \mu \nu}^{b d}(0) G_{2 \kappa}^{\mu e m}(x-y)\right. \\
& +G_{3 \nu \rho \kappa}^{a c m}(0, x-y) G_{1}^{\rho b}(x)+G_{2 \nu \rho}^{e b}(0) G_{2 \kappa}^{\rho d m}(x-y)+G_{2 \nu \rho}^{d e}(0) G_{2 \kappa}^{\rho b m}(x-y)+G_{1}^{\mu b}(x) G_{3 \mu \nu \kappa}^{d e m}(0, x-y) \\
& \left.+G_{2 \kappa}^{\mu b m}(x-y) G_{1 \mu}^{d}(x) G_{1 \nu}^{e}(x)+G_{1}^{\mu b}(x) G_{2 \mu \kappa}^{d m}(x-y) G_{1 \nu}^{e}(x)+G_{1}^{\mu b}(x) G_{1 \mu}^{d}(x) G_{2 \nu \kappa}^{e m}(x-y)\right) \\
& \left.=g f^{a b c}\left(\partial_{\nu} K_{3 \kappa}^{b c m}(0, x-y)+\partial_{\nu}\left(\bar{P}_{1}^{b}(x) K_{2 \kappa}^{c m}(x-y)\right)\right)+\partial_{\nu}\left(\bar{K}_{2 \kappa}^{b m}(x-y) P_{1}^{c}(x)\right)\right)+\delta_{a m} g_{\nu \kappa} \delta^{4}(x-y) .
\end{aligned}
$$

These corrections are completely irrelevant for the conclusions of the paper, as the omitted term in the equation for the 2-point function is just another term for the mass correction that adds to the two others already present. 